\title{
MARCINKIEWICZ-TYPE STRONG LAW OF LARGE NUMBERS FOR DOUBLE ARRAYS OF PAIRWISE INDEPENDENT RANDOM VARIABLES
}

\author{
DUG HUN HONG and SEOK YOON HWANG
}

(Received 31 May 1996)

ABSTRACT. Let $\left\{X_{i j}\right\}$ be a double sequence of pairwise independent random variables. If $P\left\{\left|X_{m n}\right| \geq t\right\} \leq P\{|X| \geq t\}$ for all nonnegative real numbers $t$ and $E|X|^{p}\left(\log ^{+}|X|\right)^{3}<\infty$, for $1<p<2$, then we prove that

$$
\frac{\sum_{i=1}^{m} \sum_{j=1}^{n}\left(X_{i j}-E X_{i j}\right)}{(m n)^{1 / p}} \rightarrow 0 \text { a.s. as } m \vee n \rightarrow \infty .
$$

Under the weak condition of $E|X|^{p} \log ^{+}|X|<\infty$, it converges to 0 in $L^{1}$. And the results can be generalized to an $r$-dimensional array of random variables under the conditions $E|X|^{p}\left(\log ^{+}|X|\right)^{r+1}<\infty, E|X|^{p}\left(\log ^{+}|X|\right)^{r-1}<\infty$, respectively, thus, extending Choi and Sung's result [1] of the one-dimensional case.

Keywords and phrases. Strong law of large numbers, pairwise independent.

1991 Mathematics Subject Classification. 60F15.

1. Introduction. Etemadi [3] extended the classical law of large numbers for i.i.d. random variables to the case where the random variables are pairwise i.i.d., i.e., if $\left\{X_{n}\right\}$ is a sequence of pairwise i.i.d. random variables with $E\left|X_{1}\right|<\infty$, then

$$
\frac{\sum_{i=1}^{n}\left(X_{i}-E X_{i}\right)}{n} \rightarrow 0 \text { a.s. }
$$

In 1985, Choi and Sung [1] have shown that if $\left\{X_{n}\right\}$ are pairwise independent and are dominated in distribution by a random variable $X$ with $E|X|^{p}\left(\log ^{+}|X|\right)^{2}<\infty, 1<$ $p<2$, then $\frac{\sum_{i=1}^{n}\left(X_{i}-E X_{i}\right)}{n^{1 / p}} \longrightarrow 0$ a.s. In addition, if $E|X|^{p}<\infty$, then it converges to 0 in $L^{1}$.

For a double sequence $\left\{X_{i j}\right\}$ of pairwise i.i.d. random variables, also Etemadi [3] proved that if $E\left|X_{11}\right| \log ^{+}\left|X_{11}\right|<\infty$, then

$$
\frac{\sum_{i=1}^{m} \sum_{j=1}^{n}\left(X_{i j}-E X_{i j}\right)}{m n} \rightarrow 0 \text { a.s. as } m \vee n \rightarrow \infty .
$$

Now, we are interested in the extension of Choi and Sung's result of the onedimensional case to a multi-dimensional array of pairwise independent random variables, which is established in the next section.

2. Main results. Let $\left\{X_{i j}\right\}$ be a double sequence of random variables and let $X_{i j}^{\prime}=$ $X_{i j} I\left\{\left|X_{i j}\right| \leq(i j)^{1 / p}\right\}, X_{i j}^{\prime \prime}=X_{i j} I\left\{\left|X_{i j}\right|>(i j)^{1 / p}\right\}$ for $1<p<2$. Throughout this paper, 
$c$ denotes an unimportant positive constant which is allowed to change and $d_{k}$ the number of all divisors of integer $k$.

To prove the main theorem, we need the following lemmas.

LEMMA 2.1. Let $\left\{X_{i j}\right\}$ be a double sequence of pairwise independent random variables. If $P\left\{\left|X_{m n}\right| \geq t\right\} \leq P\{|X| \geq t\}$ for all nonnegative real numbers $t$, then

(a)

$$
\sum_{i=1}^{\infty} \sum_{j=1}^{\infty} \frac{E\left|X_{i j}^{\prime}\right|^{2}}{(i j)^{2 / p}} \leq c E|X|^{p} \log ^{+}|X|,
$$

(b)

$$
\sum_{i=1}^{\infty} \sum_{j=1}^{\infty} \frac{E\left|X_{i j}^{\prime \prime}\right|}{(i j)^{1 / p}} \leq c E|X|^{p} \log ^{+}|X| \text { for } 1<p<2 .
$$

Proof. The estimation of $E\left|X_{i j}^{\prime}\right|^{2}$ is given by

$$
\begin{aligned}
E\left|X_{i j}^{\prime}\right|^{2} & \leq \int_{0}^{(i j)^{2 / p}} P\left(\left|X_{i j}\right|^{2} \geq t\right) d t \\
& \leq \int_{0}^{(i j)^{2 / p}} P\left(|X|^{2} \geq t\right) d t \\
& =\int_{0}^{(i j)^{2 / p}}\left\{P\left(t \leq|X|^{2}<(i j)^{2 / p}\right)+P\left((i j)^{2 / p} \leq|X|^{2}\right)\right\} d t \\
& =\int_{0}^{(i j)^{1 / p}} x^{2} d F(x)+(i j)^{2 / p} P\left((i j)^{2 / p} \leq|X|^{2}\right),
\end{aligned}
$$

where $F(x)$ is the distribution of $X$. If we use the fact that $\sum_{k=i+1}^{\infty} d_{k} / k^{2 / p}=O(\log i /$ $\left.(i+1)^{2 / p-1}\right)$, we obtain

$$
\begin{aligned}
\sum_{i=1}^{\infty} \sum_{j=1}^{\infty} \frac{1}{(i j)^{2 / p}} \int_{0}^{(i j)^{1 / p}} x^{2} d F(x) & \leq c \sum_{k=1}^{\infty} \frac{d_{k}}{k^{2 / p}} \int_{0}^{k^{1 / p}} x^{2} d F(x) \\
& \leq c \sum_{i=0}^{\infty}\left(\sum_{k=i+1}^{\infty} \frac{d_{k}}{k^{2 / p}}\right) \int_{i^{1 / p}}^{(i+1)^{1 / p}} x^{2} d F(x) \\
& \leq c \sum_{i=0}^{\infty} \frac{\log i}{(i+1)^{2 / p-1}} \int_{i^{1 / p}}^{(i+1)^{1 / p}} x^{2} d F(x) \\
& \leq c E|X|^{p} \log ^{+}|X|<\infty
\end{aligned}
$$

And

$$
\begin{aligned}
\sum_{i=1}^{\infty} \sum_{j=1}^{\infty} P\left((i j)^{2 / p} \leq|X|^{2}\right) & =\sum_{k=1}^{\infty} d_{k} P\left(k \leq|X|^{p}\right) \\
& =\sum_{k=1}^{\infty}\left(\sum_{j=1}^{k} d_{j}\right) P\left(k \leq|X|^{p}<k+1\right) \\
& =c \sum_{k=1}^{\infty} k \log k P\left(k \leq|X|^{p}<k+1\right) \\
& \leq c E|X|^{p} \log ^{+}|X|<\infty
\end{aligned}
$$


where we use the fact that $\sum_{k=1}^{n} d_{k}=O(n \log n)$. It follows that

$$
\sum_{i=1}^{\infty} \sum_{j=1}^{\infty} \frac{E\left|X_{i j}^{\prime}\right|^{2}}{(i j)^{2 / p}}<\infty, \quad \text { which proves (a). }
$$

By the fact that $\sum_{k=1}^{n} d_{k} / k^{1 / p}=O\left(n^{1-(1 / p)} \log n\right)$, we can obtain (b) by the same method.

The following lemma is a two parameter analog of [5, Lem. 3.6.1a].

LEMMA 2.2. Let $\left\{X_{i j}\right\}$ be a double sequence of pairwise independent random variables with $E X_{i j}=0$, and let $S_{m n}=\sum_{i=1}^{m} \sum_{j=1}^{n} X_{i j}$. Then

$$
E\left(\max _{\substack{1 \leq i \leq m \\ 1 \leq j \leq n}}\left|S_{i j}\right|\right)^{2} \leq c(\log m)^{2}(\log n)^{2} \sum_{k=1}^{m} \sum_{l=1}^{n} E\left|X_{k l}\right|^{2} .
$$

Proof. For $m=1$ and $n=1$, the inequality is trivial. If $m>1$, let $s$ be an integer such that $2^{s-1}<m \leq 2^{s}$. And if $n>1$, let $t$ be an integer such that $2^{t-1}<n \leq 2^{t}$. We can assume that $m, n>1$. We assign $X_{i j}$ to the point $(i, j)$ of integer in $\left(0,2^{s}\right] \times\left(0,2^{t}\right]$ (if $m<i \leq 2^{s}$ or $n<j \leq 2^{t}$, set $\left.X_{i j}=0\right)$. Divide the interval $\left(0,2^{s}\right]$ into $\left(0,2^{s-1}\right]$ and $\left(2^{s-1}, 2^{s}\right.$, each of these two intervals into two halves, and so on. Then the elements of the $i$ th partition are of length $2^{s-i}, i=0, \ldots, s$. Also, divide the interval $\left(0,2^{t}\right]$ in the same way. Then we obtain the $(i, j)$ th partition $P_{i j}$ of $\left(0,2^{s}\right] \times\left(0,2^{t}\right]$ by the $i$ th partition of $\left(0,2^{s}\right]$ and the $j$ th partition of $\left(0,2^{t}\right]$. Every rectangle $(0, i] \times(0, j]$ is the sum of at most $(s+1)(t+1)$ disjoint subrectangles each of which belongs to a different partition. We can write $S_{i j}=\sum_{k=0}^{s} \sum_{l=0}^{t} Y_{k l ; i j}$, where $Y_{k l ; i j}$ is the sum of all r.v.'s belonging to the rectangle $(a, b] \times(c, d], b-a=2^{k}$ and $d-c=2^{l}$, which may or may not be a summand of $(0, i] \times(0, j]$ so that some $Y_{k l ; i j}$ may vanish. Let $T_{i j}=$ $\sum_{k=1}^{2^{i}} \sum_{l=1}^{2^{j}}\left|Y_{k l}\right|^{2}$, where $Y_{k l}$ is the sum of all r.v.'s which belong to the $(k, l)$-element of $P_{i j}$. If we put $T=\sum_{i=0}^{s} \sum_{j=0}^{t} T_{i j}$, by the elementary Schwarz inequality, we obtain

$$
\left|S_{i j}\right|^{2} \leq(s+1)(t+1) \sum_{k=0}^{s} \sum_{l=0}^{t}\left|Y_{k l ; i j}\right|^{2} \leq(s+1)(t+1) T .
$$

Since $E T_{i j} \leq \sum_{k=1}^{m} \sum_{l=1}^{n} E\left|X_{k l}\right|^{2}, E T \leq(s+1)(t+1) \sum_{k=1}^{m} \sum_{l=1}^{n} E\left|X_{k l}\right|^{2}$. It follows that

$$
\begin{aligned}
E\left(\max _{\substack{1 \leq i \leq m \\
1 \leq j \leq n}}\left|S_{i j}\right|^{2}\right) & \leq(s+1)^{2}(t+1)^{2} \sum_{k=1}^{m} \sum_{l=1}^{n} E\left|X_{k l}\right|^{2} \\
& \leq c(\log m)^{2}(\log n)^{2} \sum_{k=1}^{m} \sum_{l=1}^{n} E\left|X_{k l}\right|^{2} .
\end{aligned}
$$

THEOREM 2.3. Let $\left\{X_{i j}\right\}$ be a double sequence of pairwise independent random variables. If $P\left\{\left|X_{m n}\right| \geq t\right\} \leq P\{|X| \geq t\}$ for all nonnegative real numbers $t$ and $E|X|^{p}\left(\log ^{+}\right.$ $|X|)^{3}<\infty$, for $1<p<2$, then

$$
\lim _{m \vee n \rightarrow \infty} \frac{\sum_{i=1}^{m} \sum_{j=1}^{n}\left(X_{i j}-E X_{i j}\right)}{(m n)^{1 / p}}=0 \text { a.s. }
$$


Proof. We denote by $S_{m n}=\sum_{i=1}^{m} \sum_{j=1}^{n} X_{i j}, S_{m n}^{\prime}=\sum_{i=1}^{m} \sum_{j=1}^{n} X_{i j}^{\prime}$. Then we obtain the inequalities

$$
\begin{aligned}
\sum_{i=1}^{\infty} \sum_{j=1}^{\infty} P\left\{X_{i j} \neq X_{i j}^{\prime}\right\} & =\sum_{k=1}^{\infty} d_{k} P\left\{\left|X_{11}\right|>k^{1 / p}\right\} \\
& \leq \sum_{k=1}^{\infty} d_{k} P\left\{|X|>k^{1 / p}\right\} \\
& =\sum_{i=1}^{\infty}\left(\sum_{k=1}^{i} d_{k}\right) \int_{i^{1 / p}}^{(i+1)^{1 / p}} d F(x) \\
& \leq c \sum_{i=1}^{\infty} i \log i \int_{i^{1 / p}}^{(i+1)^{1 / p}} d F(x) \\
& \leq c E|X|^{p} \log ^{+}|X|<\infty,
\end{aligned}
$$

Hence, by the Borel-Cantelli lemma,

$$
\frac{\sum_{i=1}^{m} \sum_{j=1}^{n}\left(X_{i j}-X_{i j}^{\prime}\right)}{(m n)^{1 / p}} \rightarrow 0 \text { a.s. }
$$

Now, we use Chebyshev's inequality and Lemma 2.1 to obtain

$$
\begin{aligned}
\sum_{k=1}^{\infty} \sum_{l=1}^{\infty} P\left\{\left|\frac{S_{2^{k} 2^{l}}^{\prime}-E S_{2^{k} 2^{l}}^{\prime}}{\left(2^{k} 2^{l}\right)^{1 / p}}\right|>\epsilon\right\} & \leq c \sum_{k=1}^{\infty} \sum_{l=1}^{\infty} \frac{\operatorname{Var} S_{2^{k} 2^{l}}^{\prime}}{\left(2^{k} 2^{l}\right)^{2 / p}} \\
& =c \sum_{k=1}^{\infty} \sum_{l=1}^{\infty} \frac{1}{\left(2^{k} 2^{l}\right)^{2 / p}} \sum_{i=1}^{2^{k}} \sum_{j=1}^{2^{l}} \operatorname{Var} X_{i j}^{\prime} \\
& \leq c \sum_{i=1}^{\infty} \sum_{j=1}^{\infty} \frac{E X_{i j}^{\prime}}{(i j)^{2 / p}} \\
& \leq c E|X|^{p} \log ^{+}|X|^{p}<\infty,
\end{aligned}
$$

which follows easily by summation by parts. It follows that

$$
\frac{S_{2^{k} 2^{l}}^{\prime}-E S_{2^{k} 2^{l}}^{\prime}}{\left(2^{k} 2^{l}\right)^{1 / p}} \longrightarrow 0 \text { a.s. }
$$

And let

$$
\begin{aligned}
T_{k l} & =\max _{\substack{2^{k} \leq m<2^{k+1} \\
2^{l} \leq n<2^{l+1}}}\left|\frac{S_{2^{k} 2^{l}}^{*}}{\left(2^{k} 2^{l}\right)^{1 / p}}-\frac{S_{m n}^{*}}{(m n)^{1 / p}}\right| \\
& \leq \frac{\left|S_{2^{k} 2^{l}}^{*}\right|}{\left(2^{k} 2^{l}\right)^{1 / p}}+\max _{\substack{2^{k} \leq m<2^{k+1} \\
2^{l} \leq n<2^{l+1}}} \frac{\left|S_{m n}^{*}\right|}{(m n)^{1 / p}},
\end{aligned}
$$

where $S_{m n}^{*}=S_{m n}^{\prime}-E S_{m n}^{\prime}$.

By using Lemma 2.2, we obtain, for any $\epsilon>0$, 


$$
\begin{aligned}
\sum_{k=0}^{\infty} \sum_{l=0}^{\infty} P\left[\max _{\substack{2^{k} \leq m \leq 2^{k+1} \\
2^{l} \leq n \leq 2^{l+1}}} \frac{\left|S_{m n}^{*}\right|}{(m n)^{1 / p}} \geq \frac{\epsilon}{2}\right] & \leq c \sum_{k=0}^{\infty} \sum_{l=0}^{\infty} \frac{1}{\left(2^{k} 2^{l}\right)^{2 / p}} E\left(\max _{\substack{2^{k} \leq m \leq 2^{k+1} \\
2^{l} \leq n \leq 2^{l+1}}}\left|S_{m n}^{*}\right|\right)^{2} \\
& \leq c \sum_{k=0}^{\infty} \sum_{l=0}^{\infty} \frac{(k+1)^{2}(l+1)^{2}}{\left(2^{2+1} 2^{l+1}\right)^{2 / p}} \sum_{i=1}^{2^{k+1}} \sum_{j=1}^{2^{l+1}} E\left|X_{i j}^{\prime}\right|^{2} \\
& \leq c \sum_{i=1}^{\infty} \sum_{j=1}^{\infty} \frac{\left(\log _{2} i j\right)^{2}}{(i j)^{2 / p}} E\left|X_{i j}^{\prime}\right|^{2}
\end{aligned}
$$

where the last inequality follows easily be summation by parts. But

$$
\begin{aligned}
\sum_{i=1}^{\infty} \sum_{j=1}^{\infty} \frac{\left(\log _{2} i j\right)^{2}}{(i j)^{2 / p}} E\left|X_{i j}^{\prime}\right|^{2} & \leq \sum_{k=1}^{\infty} \frac{d_{k}\left(\log _{2} k\right)^{2}}{k^{2 / p}} \int_{0}^{k^{1 / p}} x^{2} d F(x) \\
& \leq \sum_{i=0}^{\infty}\left(\sum_{k=i+1}^{\infty} \frac{d_{k}\left(\log _{2} k\right)^{2}}{k^{2 / p}}\right) \int_{i^{1 / p}}^{(i+1)^{1 / p}} x^{2} d F(x) \\
& \leq c \sum_{i=0}^{\infty} i^{1-(2 / p)}(\log i)^{3} \int_{i^{1 / p}}^{(i+1)^{1 / p}} x^{2} d F(x) \\
& \leq c E|X|^{p}\left(\log ^{+}|X|^{p}\right)^{3}<\infty,
\end{aligned}
$$

where we use $\sum_{k=1}^{\infty} \frac{d_{k}\left(\log _{2} k\right)^{2}}{k^{2 / p}}=O\left(\frac{(\log i)^{3}}{i^{(2 / p)-1}}\right)$ which follows by summation by parts. Hence, (2.13), (2.15), and (2.16) give us

$$
\frac{S_{m n}^{\prime}-E S_{m n}^{\prime}}{(m n)^{1 / p}} \longrightarrow 0 \quad \text { a.s. }
$$

Combining (2.11) and (2.17), we get

$$
\frac{S_{m n}-E S_{m n}^{\prime}}{(m n)^{1 / p}} \longrightarrow 0 \quad \text { a.s. }
$$

Since

$$
\frac{S_{m n}-E S_{m n}}{(m n)^{1 / p}}=\frac{S_{m n}-E S_{m n}^{\prime}}{(m n)^{1 / p}}-\frac{\sum_{i=1}^{m} \sum_{j=1}^{n} E\left|X_{i j}^{\prime \prime}\right|}{(m n)^{1 / p}},
$$

it remains to prove that the second term of the right-hand side converges to 0 a.s. By Lemma 2.1(b), we obtain

$$
\begin{aligned}
\sum_{k=1}^{\infty} \sum_{l=1}^{\infty} \frac{\sum_{i=1}^{2^{k}} \sum_{j=1}^{2^{l}} E\left|X_{i j}^{\prime \prime}\right|}{\left(2^{k} 2^{l}\right)^{1 / p}} & \leq c \sum_{i, j=1}^{\infty} \frac{E\left|X_{i j}^{\prime \prime}\right|}{(i j)^{1 / p}} \\
& \leq c E|X|^{p} \log ^{+}|x|<\infty
\end{aligned}
$$

from which, it follows that

$$
\lim _{k \vee l \rightarrow \infty} \frac{\sum_{i=1}^{2^{k}} \sum_{j=1}^{2^{l}} E\left|X_{i j}^{\prime \prime}\right|}{\left(2^{k} 2^{l}\right)^{1 / p}}=0 .
$$


But since

$$
\begin{aligned}
T_{k l}^{\prime} & =\max _{\substack{2^{k} \leq m \leq 2^{k+1} \\
2^{l} \leq n \leq 2^{l+1}}}\left|\frac{\sum_{i=1}^{m} \sum_{j=1}^{n} E\left|X_{i j}^{\prime \prime}\right|}{(m n)^{1 / p}}-\frac{\sum_{i=1}^{2^{k}} \sum_{j=1}^{2^{l}} E\left|X_{i j}^{\prime \prime}\right|}{\left(2^{k} 2^{l}\right)^{1 / p}}\right| \\
& \leq \frac{c}{\left(2^{k+1} 2^{l+1}\right)^{1 / p}} \sum_{i=1}^{2^{k+1}} \sum_{j=1}^{2^{l+1}} E\left|X_{i j}^{\prime \prime}\right|,
\end{aligned}
$$

$T_{k l}^{\prime}$ converges to 0 which implies that, by (2.21),

$$
\frac{\sum_{i=1}^{m} \sum_{j=1}^{n} E\left|X_{i j}^{\prime \prime}\right|}{(m n)^{1 / p}} \rightarrow 0 \text {. }
$$

This completes the proof.

COROLlary 2.4. Let $\left\{X_{i j}\right\}$ be a double sequence of pairwise i.i.d. random variables with $E\left|X_{11}\right|^{p}\left(\log ^{+}\left|X_{11}\right|\right)^{3}<\infty$, for $1<p<2$. Then

$$
\lim _{m \vee n \rightarrow \infty} \frac{\sum_{i=1}^{m} \sum_{j=1}^{n}\left(X_{i j}-E X_{i j}\right)}{(m n)^{1 / p}}=0 \text { a.s. }
$$

REMARK. The generalization to $r$-dimensional arrays of random variables can be obtained easily under the condition $E|X|^{p}\left(\log ^{+}|X|\right)^{r+1}<\infty$.

THEOREM 2.5. Let $\left\{X_{i j}\right\}$ be a double sequence of pairwise independent random variables. If $P\left\{\left|X_{i j}\right| \geq t\right\} \leq P\{|X| \geq t\}$ for all nonnegative real numbers $t$ and $E|X|^{p} \log ^{+}$ $|X|<\infty, 1<p<2$, then

$$
\frac{\sum_{i=1}^{m} \sum_{j=1}^{n}\left(X_{i j}-E X_{i j}\right)}{(m n)^{1 / p}} \longrightarrow 0 \quad \text { in } L^{1} \text { as } m \vee n \rightarrow \infty .
$$

Proof. Since $\left\{X_{i j}\right\}$ are pairwise independent, $\left\{X_{i j}^{\prime}-E X_{i j}^{\prime}\right\}$ are orthogonal which implies that

$$
E\left|\frac{\sum_{i=1}^{m} \sum_{j=1}^{n}\left(X_{i j}^{\prime}-E X_{i j}^{\prime}\right)}{(m n)^{1 / p}}\right|^{2} \leq \frac{\sum_{i=1}^{m} \sum_{j=1}^{n} E\left|X_{i j}^{\prime}\right|^{2}}{(m n)^{2 / p}} .
$$

Since

$$
\begin{aligned}
E\left|\frac{\sum_{i=1}^{m} \sum_{j=1}^{n}\left(X_{i j}-E X_{i j}\right)}{(m n)^{1 / p}}\right| \leq & E\left|\frac{\sum_{i=1}^{m} \sum_{j=1}^{n}\left(X_{i j}^{\prime}-E X_{i j}^{\prime}\right)}{(m n)^{1 / p}}\right| \\
& +2 \frac{\sum_{i=1}^{m} \sum_{j=1}^{n} E\left|X_{i j}^{\prime \prime}\right|}{(m n)^{1 / p}}
\end{aligned}
$$

it suffices to show that $\left(\sum_{i=1}^{m} \sum_{j=1}^{n} E\left|X_{i j}^{\prime}\right|^{2}\right) /(m n)^{2 / p}$ converges to 0 as $m \vee n \rightarrow 0$. But this can be shown by a method similar to that used in the proof of (2.23) in Theorem 2.3.

COROLLARY 2.6. Let $\left\{X_{i j}\right\}$ be a double sequence of pairwise i.i.d. random variable with $E\left|X_{11}\right|^{p} \log ^{+}\left|X_{11}\right|<\infty$, for $1<p<2$. Then

$$
\frac{\sum_{i=1}^{m} \sum_{j=1}^{n}\left(X_{i j}-E X_{i j}\right)}{(m n)^{1 / p}} \rightarrow 0 \text { in } L^{1} \text { as } m \vee n \rightarrow \infty .
$$


REMARK. The generalization to $r$-dimensional arrays of random variables can be obtained under the condition $E|X|^{p}\left(\log ^{+}|X|\right)^{r+1}<\infty$.

ACKNOWLEDGEMENT. This research was supported by the Catholic University of Taegu-Hyosung.

\section{REFERENCES}

[1] B. D. Choi and S. H. Sung, On convergence of $\left(S_{n}-E S_{n}\right) / n^{1 / r}, 1<r<2$, for pairwise independent random variables, Bull. Korean Math. Soc. 22 (1985), no. 2, 79-82. MR 87f:60044. Zbl 585.60043.

[2] K. L. Chung, A course in probability theory, second ed., Probability and Mathematical Statistics, vol. 21, Academic Press, New York, 1974. MR 49 11579. Zbl 345.60003.

[3] N. Etemadi, An elementary proof of the strong law of large numbers, Z. Wahrsch. Verw. Gebiete 55 (1981), no. 1, 119-122. MR 82b:60027. Zbl 448.60024.

[4] G. H. Hardy and E. M. Wright, An introduction to the theory of numbers, fourth ed., Clarendon Press, Oxford, 1960. Zbl 086.25803.

[5] M. Loeve, Probability theory. I, fourth ed., Graduate Texts in Mathematics, vol. 45, SpringerVerlag, New York, 1977. MR 58 31324a. Zbl 359.60001.

[6] R. T. Smythe, Strong laws of large numbers for $r$-dimensional arrays of random variables, Ann. Probability 1 (1973), no. 1, 164-170. MR 49 11602. Zbl 258.60026.

[7] W. F. Stout, Almost sure convergence, Probability and Mathematical Statistics, vol. 24, Academic Press, New York, 1974. MR 56 13334. Zbl 321.60022.

Hong: School of Mechanical and Automotive Engineering, CATHOlic UNIVERSiTy of TAEgu-Hyosung, KyUngBuK 712-702, SOUTH KoreA

HWANG: DePARTMENT OF MATHEMATICS, TAEgu UNIVERSITY, KyUnGbUK 713-714, SOUTH KoREA 


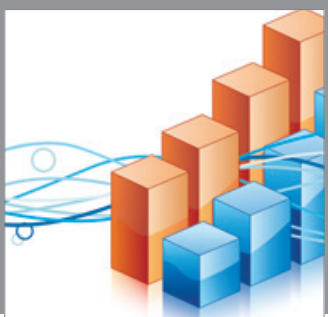

Advances in

Operations Research

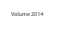

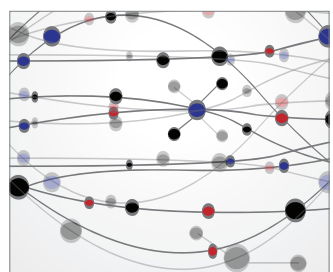

\section{The Scientific} World Journal
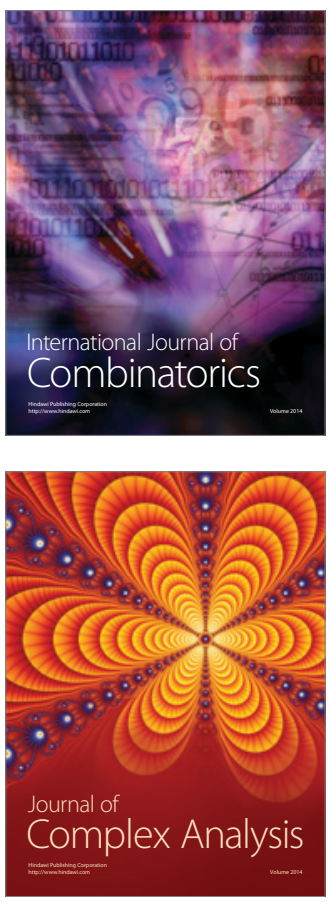

International Journal of

Mathematics and

Mathematical

Sciences
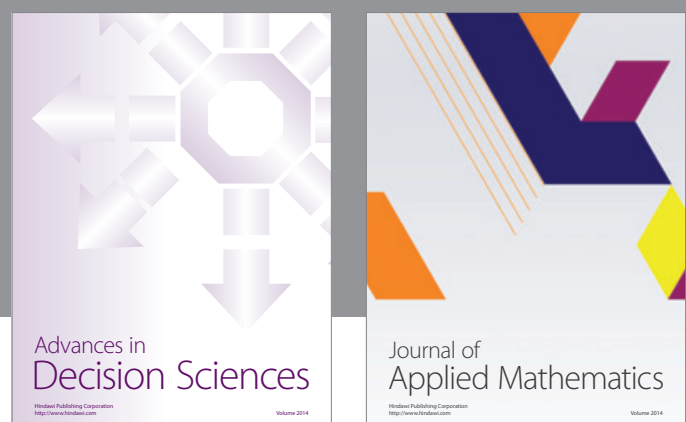

Journal of

Applied Mathematics
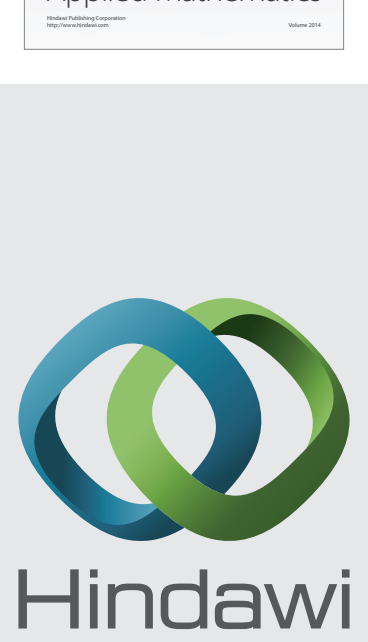

Submit your manuscripts at http://www.hindawi.com
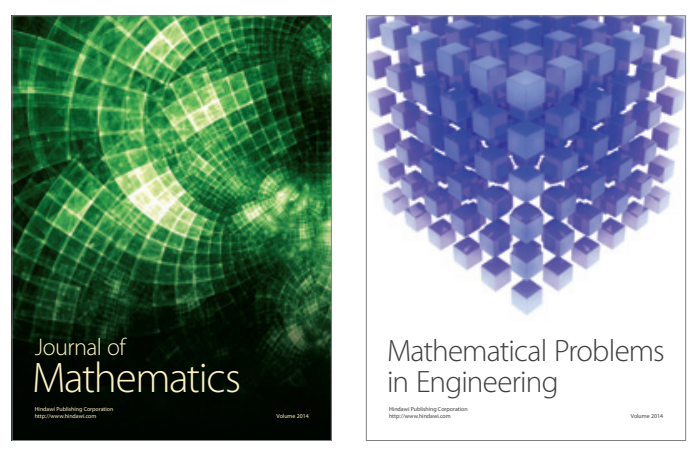

Mathematical Problems in Engineering
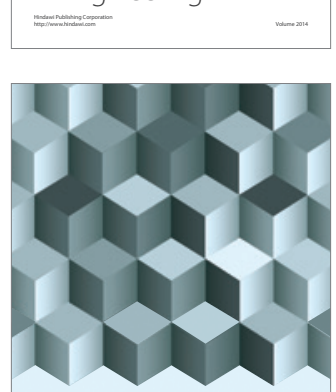

Journal of

Function Spaces
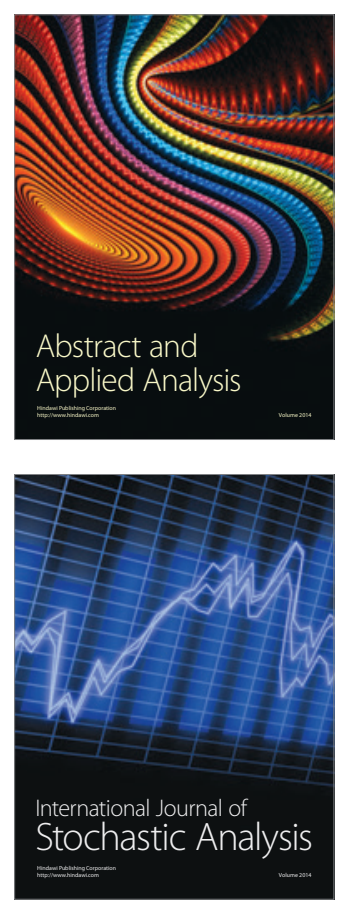

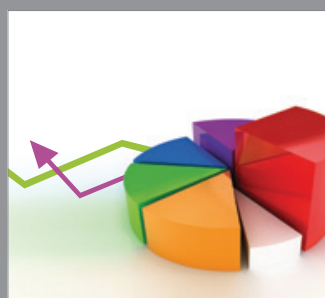

ournal of

Probability and Statistics

Promensencen
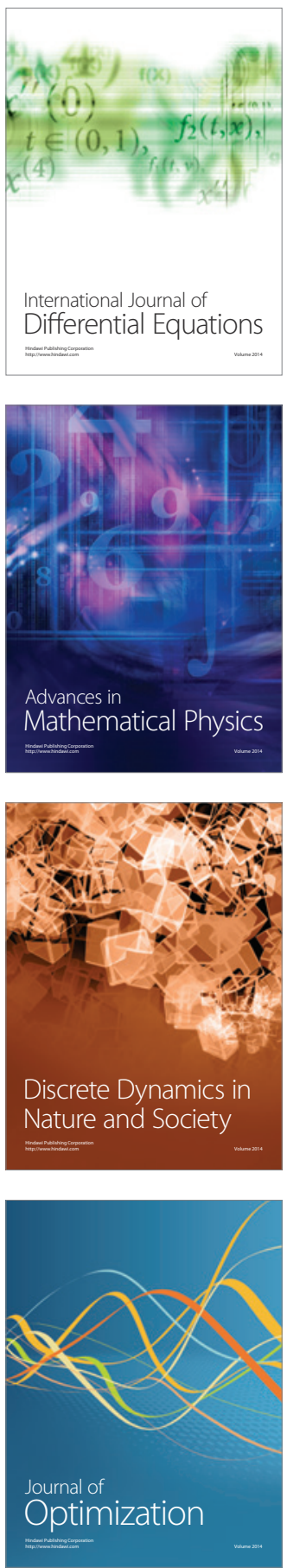